Supporting Information

\title{
Controlling the Spreading of Nanoliter Scale Droplets on the Fiber of Fabrics for Enhancing Image Quality and Ink Utilization
}

Kun Zhang a, b, c, Kuanjun Fang a, b, c, *, Weichao Chen a, b, c, Fuyun Sun d, Yawei Song, a, b, c, Lei Han ${ }^{\text {a, b, c }}$

${ }^{\text {a }}$ College of Textiles \& Clothing, Qingdao University, 308 Ningxia Road, Qingdao 266071, China

b State Key Laboratory for Biofibers and Eco-textiles, 308 Ningxia Road, Qingdao 266071, China

c Collaborative Innovation Center for Eco-textiles of Shandong Province and the Ministry of Education, 308 Ningxia Road, Qingdao 266071, China

d YuYue Home Textile Company, 1 Xiner Road, Bincheng District, Binzhou, 256600, China

* Corresponding author. Qingdao University, 308 Ningxia Road, Qingdao 266071, China.

E-mail address: 13808980221@163.com (K. Fang). 


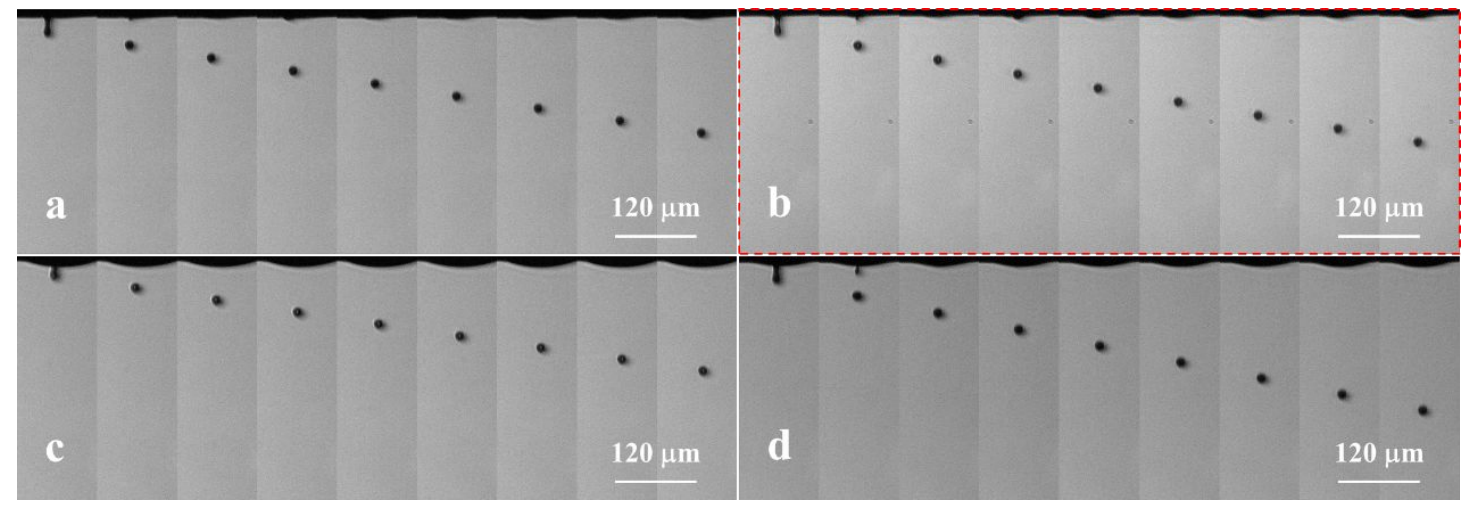

Figure S1. Droplet formations of (a) RB49 solution, (b) RR218 solution, (c) RY95 solution and (d)

RB8. The jetting frequency was $710 \mathrm{~Hz}$. The time interval between frames was $20 \mu \mathrm{s}$.

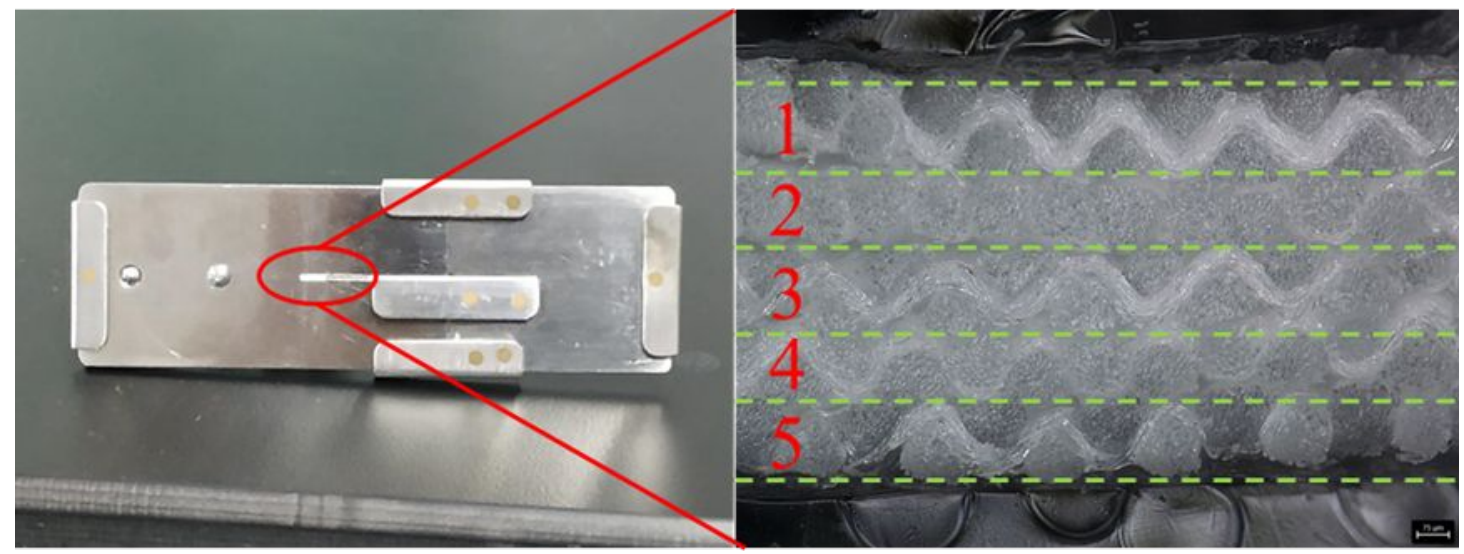

Figure S2. Schematic graph of making the cotton fabric slice. 


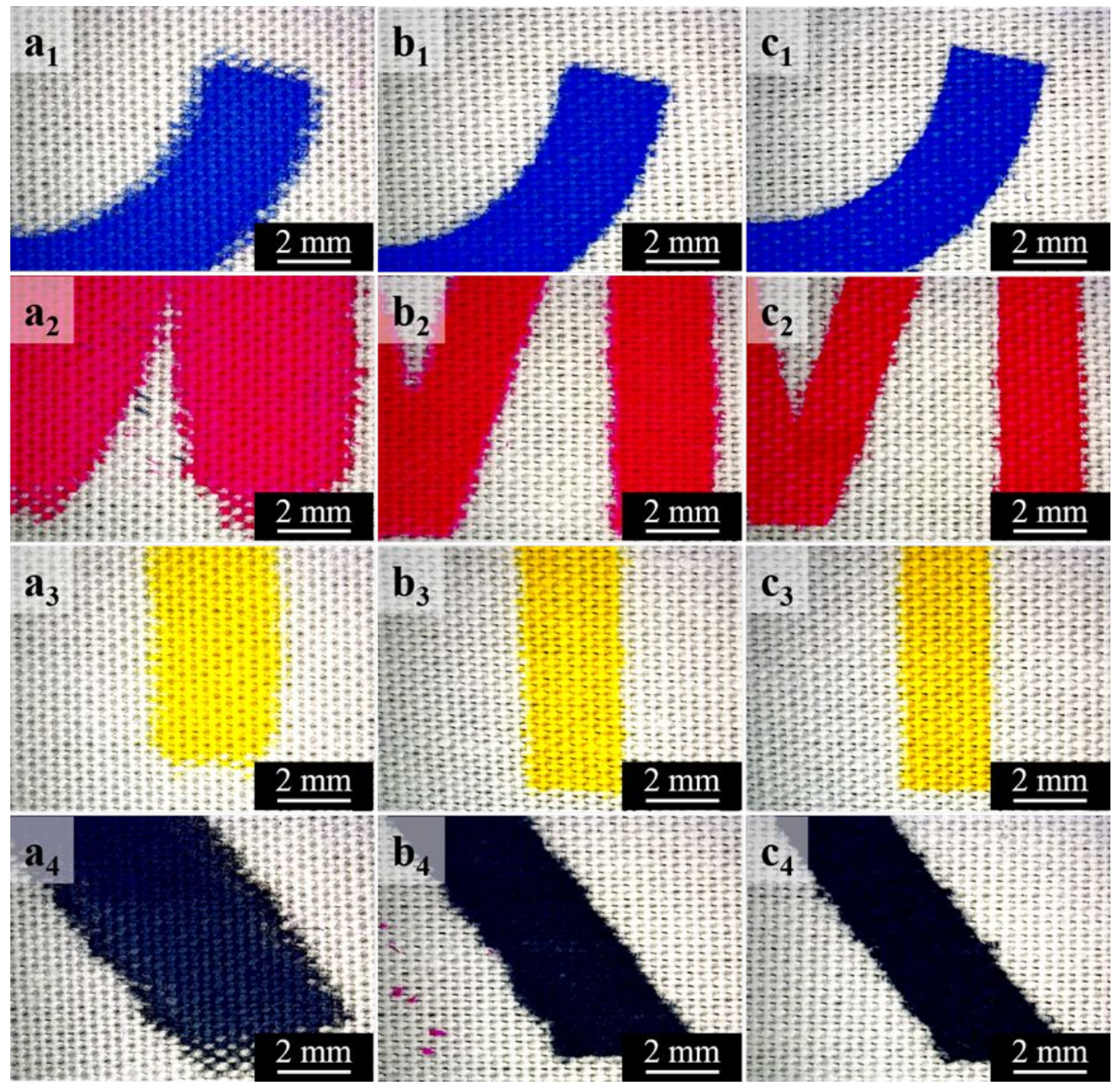

Figure S3. Magnified images of the printed (1) C, (2) M, (3) Y and (4) K on (a) $C_{U N}$, (b) $C_{H E C}$ and (c) $\mathrm{C}_{\mathrm{HPMC}}$.
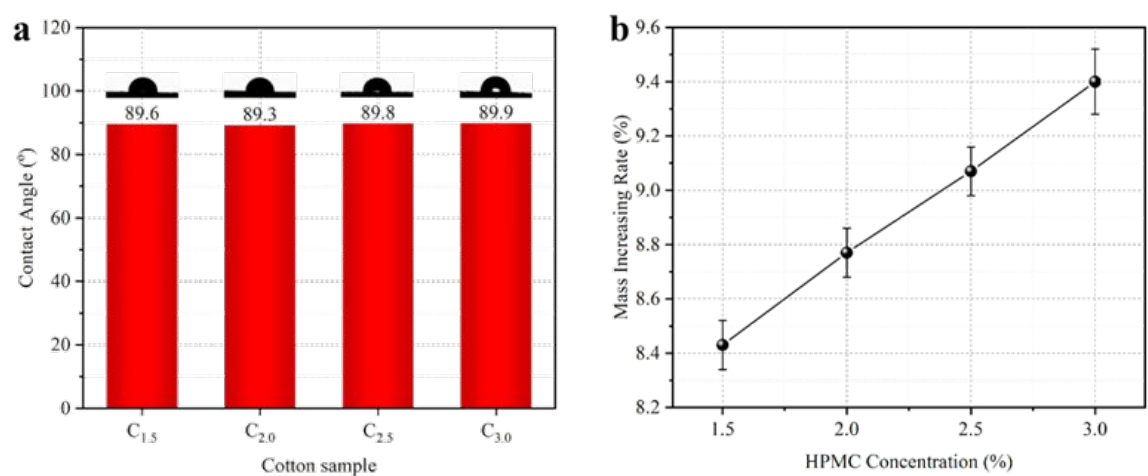

Figure S4. (a) contact angles of a $2 \mu \mathrm{L}$ of water droplet on the fabrics treated with HPMC of different concentrations. (b) mass increasing rates of the fabrics treated with HPMC of different concentrations. 
Table S1. The rubbing fastnesses of cyan, magenta, yellow and black colors on the fabrics

\begin{tabular}{cccccccccc}
\hline & \multicolumn{8}{c}{ Rubbing fastness } \\
\cline { 2 - 9 } Fabric sample & \multicolumn{8}{c}{ dry } & \multicolumn{5}{c}{ wet } \\
\cline { 2 - 9 } & cyan & magenta & yellow & black & cyan & magenta & yellow & black \\
\hline $\mathrm{C}_{\mathrm{UN}}$ & 5 & $4-5$ & $4-5$ & 5 & $4-5$ & $4-5$ & $4-5$ & $4-5$ \\
$\mathrm{C}_{\text {HEC }}$ & 5 & $4-5$ & $4-5$ & 5 & $4-5$ & $4-5$ & $4-5$ & $4-5$ \\
$\mathrm{C}_{\text {HPMC }}$ & 5 & $4-5$ & $4-5$ & 5 & $4-5$ & $4-5$ & 4 & $4-5$ \\
\hline
\end{tabular}

Table S2. The washing fastnesses of cyan, magenta, yellow and black colors on the fabrics

\begin{tabular}{cccccccccc}
\hline & \multicolumn{8}{c}{ Washing fastness } \\
\cline { 2 - 8 } Fabric sample & \multicolumn{8}{c}{ Staining to cotton fabrics } & \multicolumn{7}{c}{ Color change } \\
\cline { 2 - 8 } & cyan & magenta & yellow & black & cyan & magenta & yellow & black \\
\hline $\mathrm{C}_{\mathrm{UN}}$ & $4-5$ & $4-5$ & $4-5$ & 5 & $4-5$ & $4-5$ & $4-5$ & 5 \\
$\mathrm{C}_{\mathrm{HEC}}$ & $4-5$ & $4-5$ & $4-5$ & 5 & $4-5$ & $4-5$ & $4-5$ & $4-5$ \\
$\mathrm{C}_{\mathrm{HPMC}}$ & $4-5$ & $4-5$ & $4-5$ & 5 & $4-5$ & $4-5$ & $4-5$ & $4-5$ \\
\hline
\end{tabular}

